\title{
EVALUACIÓN DE TRAZADORES QUÍMICOS EN UN FLUIDO DE FRACTURA BASE AGUA GOMA GUAR
}

\author{
José luis Hernández Parra*1; Zarith del Pilar Pachón Contreras²; Zuly Himelda Calderón Carrillo ${ }^{3}$ \\ * A quien debe dirigirse la correspondencia.
}

\begin{abstract}
RESUMEN
En esta investigación, se evaluaron siete sustancias químicas seleccionadas de las familias de sales inorgánicas y colorantes usados normalmente en hidrología, como potenciales trazadores para un fracturamiento hidráulico, implementando métodos analíticos económicos para su detección y cuantificación y que se puedan realizarse in situ. A partir de los ensayos de adsorción, compatibilidad con el gel de fractura y los fluidos de formación, así como ensayos de desplazamiento en medio poroso, se identificaron tres sustancias que respondieron aceptablemente, tras ser sometidas a las condiciones físicas y químicas de un proceso de fractura hidráulica lo que las habilitaría para su uso como trazadores.
\end{abstract}

Palabras clave: Fracturamiento Hidráulico, Fluido de Fractura, Flowback, Polímero, Trazadores Químicos, Colorantes, Adsorción, Desplazamiento en medio poroso.

\section{EVALUATION OF CHEMICAL TRACERS IN FRACTURING FLUID BASED IN WATER GUAR}

\begin{abstract}
In this research, seven chemicals substances selected from the families of inorganic salts and dyes commonly used in hydrology were evaluated as potential tracers for hydraulic fracturing using economic analytical methods implemented for their detection and quantification and that also can be performed in situ. From adsorption tests, compatibility between the fracture gel and formation fluids and core flooding tests, three substances responded acceptably, after being subjected to physical and chemical conditions of a hydraulic fracturing process which enable them to be used as tracers.
\end{abstract}

Keywords: Hydraulic Fracturing, Fracturing Fluid, Flowback, Polymer, Chemical Tracers, Dyes, Adsorption, Core Flooding.

1. Escuela de Ingeniería de Petróleos, Universidad Industrial de Santander, UIS, Carrera 27 calle 9, Bucaramanga, Colombia. E-mail: j1181ph@hotmail.com

2. Instituto Colombiano del Petróleo, ICP. Kilómetro 7 vía Bucaramanga - Piedecuesta, Piedecuesta, Colombia. E-mail: zarith.pachon@ecopetrol.com.co

3. Escuela de Ingeniería de Petróleos, Universidad Industrial de Santander, UIS, Carrera 27 calle 9, Bucaramanga, Colombia. E-mail: calderon@uis.edu.co 


\section{INTRODUCCIÓN}

Un número significativo de pozos de gas y aceite son hidráulicamente fracturados con el objetivo de maximizar el índice de productividad (IP) en los pozos. Sin embargo, no siempre se obtienen los resultados esperados, en gran medida por el residuo polimérico retenido en el medio poroso, lo que se refleja en una baja eficiencia de retorno del fluido de fractura (FF) a superficie (flowback). ${ }^{[15]}$ Como consecuencia, la conductividad de fractura se ve reducida y su limpieza (clean up) resulta deficiente.

El método tradicional para cuantificar la limpieza de la fractura ha sido contabilizar la carga del agua durante el flujo de retorno. No obstante, este valor se ve altamente influenciado por el volumen de agua aportado por la formación. Además Este método proporciona información del recobro total de fluidos, más no del fluido de fractura retornado de cada etapa.

La literatura presenta diferentes trabajos enfocados a tener una mayor compresión de la limpieza del fluido de fractura, como el realizado por Pope et al, ${ }^{[11]}$ quienes describen una técnica colorimétrica para cuantificar el flowback, midiendo la concentración del polímero en las muestras de los retornos, pero este método es complicado y los resultados no son concluyentes. Posteriormente, Yang and Flippen ${ }^{[17]}$ determinando el contenido total de carbohidratos antes y después del tratamiento calcularon la carga total recuperada, lo cual es una medida potencial del daño por polímero.

En estudios similares Asadi y Woodroof, ${ }^{[3]}$ Sullivan, et al., ${ }^{[14]}$ y Asadi et al., ${ }^{[4]}$ han utilizado la técnica de trazadores químicos para calcular el flowback en cada segmento o etapa, lo cual permite calcular la eficiencia total. Estos estudios muestran, que la última década se ha impulsado la aplicación de los trazadores químicos como método para cuantificar la cantidad de tratamiento retenido, diagnosticar la eficiencia de limpieza y disponer de herramientas para mejorar el diseño de los fluidos de fractura o modificar las etapas de fracturamiento.

Los trazadores químicos pueden ser aplicados en cada etapa individual del proceso de fractura a una concentración conocida, una vez se inicia el proceso de flowback, las muestras son recolectadas para analizar la concentración del trazador ${ }^{[16,5]}$.

Para asegurar el éxito técnico y económico del proceso del cálculo del flowback mediante la técnica de trazadores químicos, los trazadores deben cumplir con características específicas tales como: no ser tóxicos, ser solubles en agua y no reaccionar entre ellos ni con la formación o con la tubería. También deben ser estables con la temperatura y en el tiempo, compatibles con los aditivos del FF y de la formación (agua, hidrocarburo), presentar mínima adsorción en la roca y con el agente propante. Estos trazadores deben ser detectables a bajas concentraciones, ambientalmente seguros y económicos. ${ }^{[5,9]}$

Con el objetivo de satisfacer los requisitos antes expuestos y seleccionar trazadores que sean fácil y rápidamente identificables, sin la necesidad de técnicas altamente especializadas y costosas, se evaluaron en laboratorio siete sustancias químicas de las familias de sales inorgánicas y colorantes, usadas normalmente en hidrología que pueden servir como potenciales trazadores.

El estudio se hizo para las condiciones del campo Tibú, Formación Barco, ubicado en la cuenca del Catatumbo, (al norte de Cúcuta, en el departamento de Norte de Santander, frontera con Venezuela) en el que se hará una campaña amplia de fracturamiento hidráulico. Los métodos analíticos implementados para la detección y cuantificación estuvieron basados en técnicas espectroscópicas. Se identificaron tres especies de trazadores que respondieron aceptablemente tras ser sometidas a las condiciones físicas y químicas de un proceso de fractura hidráulica lo que las habilitaría para su uso como trazadores.

\section{MARCO TEÓRICO}

La técnica de fracturamiento hidráulico consiste en el bombeo de un fluido de fractura en el yacimiento o formación, a alta presión y velocidad suficiente con el fin de fracturar la roca productora de hidrocarburo. Una parte del fluido abre la fractura y la otra invade las vecindades de la misma. Inicialmente, si la operación es exitosa, solo se inyecta fluido, como resultado se generan nuevos canales o fracturas de alta permeabilidad que son empacadas con un agente propante para evitar su cierre.

El fluido de fractura es una suspensión polimérica de tipo coloidal, que coagula adquiriendo cierta rigidez y elasticidad. El fluido base es generalmente agua pero también puede ser aceite, al cual se adiciona polímeros que tienen como efecto el incremento de la viscosidad. ${ }^{[13]}$ Sus funciones son básicamente abrir y extender la fractura, es decir crear geometría de fractura (canales), mantener en suspensión y transportar el propante (arena 
sintética) a través de la tubería, hasta distribuirlo a lo largo de la fractura creada. Al fluido base se le suman una variedad de aditivos que ayudan a conseguir las propiedades reológicas deseadas.

Una vez finalizada la operación de fractura, el fluido inyectado debe disminuir su viscosidad "FF roto" para ser retornado a superficie. Un retorno incompleto del fluido significa obstrucciones en la fractura o canal de drenaje creado en el yacimiento y por lo tanto no alcanza el objetivo de aumentar la producción. La limpieza del FF se relaciona con el daño por la cantidad de residuo polimérico retenido en la fractura después de que ésta se cierra y el que queda en la cara de la formación o roca fracturada y que se convierte en un gel de alta concentración, denominado filter cake ${ }^{[12]}$ (Figura 1).

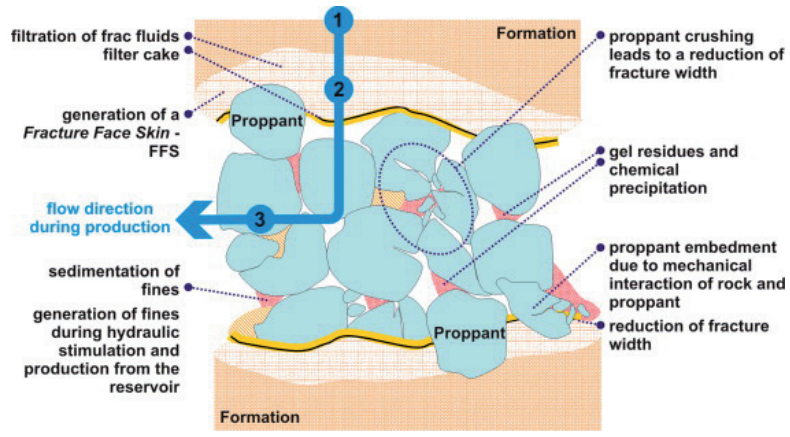

Figura 1. Clases de daños en la fractura. ${ }^{[12]}$

Recientemente, los trazadores son utilizados en un esfuerzo para reforzar el nivel de entendimiento con respecto a la dinámica de la colocación de los fluidos de fractura, lo que favorece la recopilación de información más acertada sobre el flowback. En este proceso, los trazadores son mezclados con el fluido a una concentración conocida, una vez se inicia el proceso de retorno del FF, las muestras son recolectadas para analizar la concentración del trazador ${ }^{[1]}$. Posteriormente, mediante balance de masa, se calcula el trazador en cada etapa; la eficiencia del flowback y la limpieza de la fractura. (Figura 2).
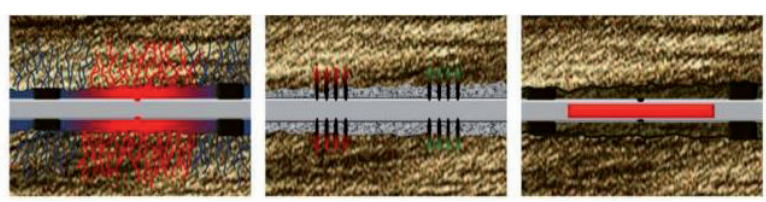

Figura 2. Esquema del Proceso de Trazado en el Fracturamiento Hidráulico ${ }^{[2]}$

Básicamente, los tipos de trazadores utilizados en la industria petrolera se dividen en trazadores radiactivos, de partición, fluorescentes, colorante y químicos. ${ }^{[8,7]}$ Cada familia posee sus propias ventajas y limitaciones.
Las restricciones y regulaciones de los primeros hacen difícil la aplicación en campo en algunas áreas. Los trazadores de partición tienen la desventaja que viajan más lentamente en comparación que el frente de avance del fluido de inyección. Los químicos como los ácidos fluorobenzóicos son rutinariamente usados en campo por algunas compañías, porque tienen un límite de detección muy bajo. Sin embargo, requieren un análisis riguroso y técnicas especializadas. ${ }^{[10,2]}$ Los marcadores fluorescentes y colorantes son de bajo costo, seguros, fácil de manipulación y detección. Su principal inconveniente es el cierto grado de degradación térmica.

\section{DESARROLLO EXPERIMENTAL}

Se evaluaron siete sustancias como posibles trazadores en FF: fluorescentes (Fluoresceína, Eosina Y), colorantes (Rojo de metilo y Rojo de fenol); iones Tiocianato y Nitrato. Para cada uno de las sustancias seleccionadas se implementó el método analítico para su cuantificación por medio de técnicas espectroscópicas.Todos ellos se sometieron a ensayos de adsorción estática con el propante y arena de referencia Ottawa (para simular la roca de yacimiento), ensayos fluido/fluido y roca/fluido, con el crudo del campo Tibú y arena de referencia Ottawa respectivamente, así como ensayos de desplazamiento en medio poroso. Estos ensayos fueron diseñados para evaluar que las sustancias químicas adicionadas no cambien las propiedades del FF por procesos de contaminación o reacciones secundarias.

\section{ENSAYOS DE ADSORCIÓN}

Cada trazador se disolvió en $250 \mathrm{ml}$ de agua destilada. Luego, a $100 \mathrm{~mL}$ de esta solución se le adicionó la mezcla de minerales constituida por $90 \%$ arena (referencia Ottawa malla 80/100) y $10 \%$ de Caolinita (mineral arcilloso presente en yacimiento). Se ajustó el $\mathrm{pH}$ de manera que fuese equivalente al del FF roto (viscosidad $<$ a $0.1 \mathrm{~Pa}$.s). La cantidad de trazador adsorbida fue cuantificada por los métodos espectrofotométricos. Todos los experimentos se realizaron a temperatura del yacimiento $\left(62^{\circ} \mathrm{C}\right)$ durante 72 horas. El procedimiento se repitió bajo las mismas condiciones pero la arena Ottawa fue reemplazada por arena natural (Brady Sand 16/30) que corresponde al propante a utilizar en los trabajos de la campaña de fracturamiento. La diferencia entre la concentración inicial y final del trazador, permite calcular la adsorción del producto sobre el material. 


\section{ENSAYOS DE COMPATIBILIDAD}

Los experimentos se realizaron con el fluido de fractura sin trazador, como referencia y dosificado con trazador para valorar su efecto. El trazador se adicionó a la formulación a partir de una solución patrón, tomado el volumen requerido de tal forma que al diluirla en 300 $\mathrm{mL}$ del FF quedase a la concentración deseada.

Las interacciones fluido-fluido incluyeron: Solubilidad, es decir la verificación de que todos los componentes del fluido de fractura y el trazador formen una sola fase acuosa al ser mezclados. La compatibilidad entre fluido de fractura roto y los fluidos de la formación (petróleo y agua), realizando mezclas en proporciones $50-50$ y $80-20 \mathrm{v} / \mathrm{v}$, agitadas manualmente por un minuto y sometidas a $62^{\circ} \mathrm{C}$ por 2 horas. Se considera que una mezcla es compatible si su separación de fases es mayor al $80 \%$ y no hay presencia de nuevas fases o precipitados. Y por último la tensión interfacial entre el FF roto y el petróleo.

Los ensayos roca-fluido correspondieron a mojabilidad visual y detergencia según norma API RP42 usando de arena de referencia Ottawa malla 20/40 y Petróleo de Tibú. La evaluación reológica de los FF se realizó según la norma API RP 13M, usando un viscosímetro Chanddler $5550 \mathrm{HP} / \mathrm{HT}$. La reología fue evaluada a la temperatura de yacimiento $\left(62^{\circ} \mathrm{C}\right), 500 \mathrm{psi}$, usando un resorte F410, rotor - Bob R1B5 y un volumen de 52 $\mathrm{mL}$. Adicionalmente, se registraron los datos: vortex time (tiempo de cierre de vórtice), crown time (tiempo de corona) y release time (tiempo de armado), como parámetros para QAQC (Quality Assurance/Quality Control).

Los ensayos de desplazamiento en medio poroso se realizaron en Bereas de baja permeabilidad, de porosidad del $18.3 \%$ y una densidad de grano de $2.67 \mathrm{~g} / \mathrm{cc}$. Se simularon fracturas abriendo una hendidura vertical de 0,3 $\mathrm{cm}$ de espesor con longitud de $4,8 \mathrm{~cm}$, correspondiente a la mitad de la longitud total de la muestra. (Figura 3). La hendidura ó fractura se rellenó con propante denominado Brady Sand 16/30. El procedimiento usado está basado en el trabajo conducido por Carrascal, Pachón \& Molina. ${ }^{[6]}$

La permeabilidad absoluta $(\mathrm{Ka})$ de la muestra fue determinada mediante desplazamiento de salmuera de $\mathrm{KCl}$ al 2\% P/V en sentido de producción (SP), las condiciones de operación fueron: (Ty: $62^{\circ} \mathrm{C}$, presión de confinamiento: 750 psi y presión de producción: 200 psi) y la velocidad de flujo $1 \mathrm{ml} / \mathrm{min}$.
Después, dos volúmenes porosos (VP) de FF dosificado con trazador fueron inyectados en sentido de inyección (SI), sin superar el diferencial de presión de 500 psi. Se dio un tiempo de cierre de 2 horas después de la inyección del fluido para garantizar rompimiento parcial del FF. Posteriormente, se desplazó salmuera de $\mathrm{KCl}$ al $2 \% \mathrm{P} / \mathrm{V}$ hasta estabilidad del diferencial de presión para determinar el retorno de permeabilidad. Los efluentes se recolectaron cada VP para determinar la concentración del trazador y del polímero.
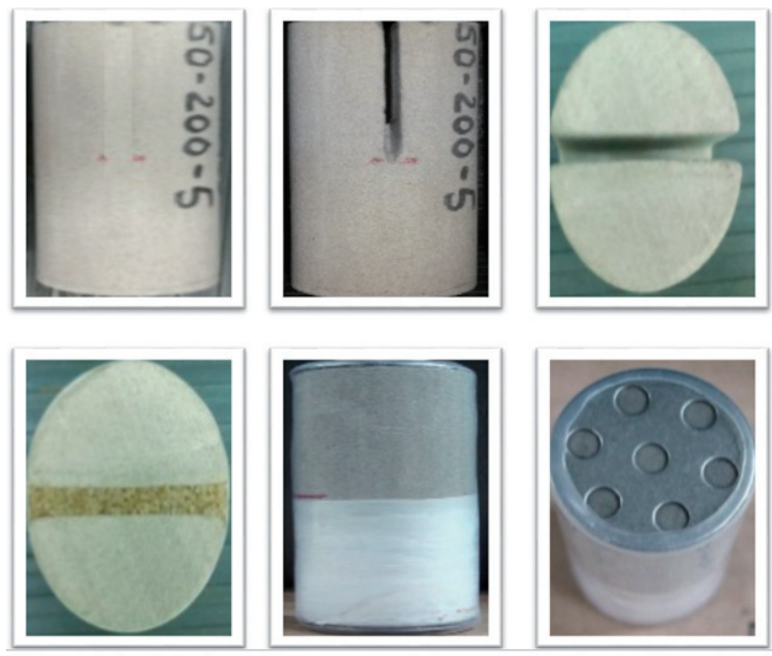

Figura 3. Preparación de los medios porosos.

Finalmente, se desplazó una solución de tratamiento post fractura para retirar del sistema poroso el polímero remanente. La concentración de los trazadores y la cantidad de polímero retenido en el sistema poroso, se determinó mediante balance de masa y siguiendo el método colorimétrico DNS (Acido 3,5 dinitrosalicílico). El cual se basa en una reacción Redox entre el Ácido 3,5 dinitrosalicílico y los azucares reductores presentes en la muestra.

\section{RESULTADOS}

Se implementaron los métodos analíticos espectroscópicos requeridos para cuantificar la concentración de las sustancias químicas evaluadas como trazadores en el FF, para ello se empleó un espectrofotómetro UV-VIS Hach DR 5000 a una longitud de onda de $420 \mathrm{~nm}$ para los nitratos, $480 \mathrm{~nm}$ para Tiocinato y $515 \mathrm{~nm}$ para rojo de metilo y rojo de fenol. Para la fluoresceína y eosina Y empleó un fluorespectrómetro Thermo Scientific NanoDrop 3300.

La linealidad de la recta de calibrado y el grado de ajuste de los puntos experimentales, en todos los casos fueron 
mayores a 0,98 indicando una relación fuerte entre las variables. La excepción fue para el rojo de fenol que no se ajustó al modelo lineal, por esta razón esta sustancia se descartó al no tener un método para cuantificarla.

\section{RESULTADOS ENSAYOS DE ADSORCIÓN}

Aunque en general los resultados descritos introducen cierta incertidumbre en la determinación del grado de adsorción, debido a que la diferencia entre la concentración inicial y final $\left(\mathrm{C}_{0}-\mathrm{C}\right)$ es muy pequeña, los resultados obtenidos permiten inferir que toda superficie tiene la misma actividad para adsorción, no hay interacción entre las especies adsorbidas; todas las adsorciones tienen lugar mediante el mismo mecanismo y el grado de adsorción es inferior a una capa monomolecular completa en la superficie, excepto los resultados obtenidos con rojo de metilo y los nitratos. (Ver Tabla 4).

Tabla 4. Adsorción estática de trazadores sobre una mezcla de minerales.

\begin{tabular}{|c|c|c|c|c|c|c|c|c|c|}
\hline \# Prueba & Trazador & Adsorbentes & $\begin{array}{c}\text { concentración } \\
(\mathrm{ppb})\end{array}$ & $\begin{array}{l}\text { Tiempo } \\
\text { de } \\
\text { prueba } \\
\text { (h) }\end{array}$ & $\begin{array}{c}\text { temperatura } \\
\left({ }^{\circ} \mathbf{F}\right)\end{array}$ & $\begin{array}{c}\text { Masa } \\
\text { inicial } \\
(\mu \mathrm{g})\end{array}$ & $\begin{array}{c}\text { Masa } \\
\text { final } \\
(\mu \mathrm{g})\end{array}$ & $\begin{array}{c}\text { Masa } \\
\text { retenida } \\
(\mu \mathrm{g})\end{array}$ & $\begin{array}{c}\text { Trazador } \\
\text { retenido } \\
(\%)\end{array}$ \\
\hline 1 & \multirow{3}{*}{ Nitratos } & \multirow{3}{*}{$\begin{array}{c}\text { Mezcla } \\
90 \% \text { Arena } \\
\text { Ottawa, } 10 \% \\
\text { de Caolinita }\end{array}$} & 400 & 60 & 143 & 99,47 & 96,8 & 2,66 & $2,7 \%$ \\
\hline 2 & & & 1500 & 60 & 143 & 354,47 & 353,1 & 1,33 & $0,4 \%$ \\
\hline 3 & & & 2500 & 60 & 143 & 588,13 & 580,8 & 7,33 & $1,2 \%$ \\
\hline 4 & \multirow{3}{*}{$\begin{array}{l}\text { Rojo de } \\
\text { Metilo }\end{array}$} & \multirow{3}{*}{$\begin{array}{c}\text { Mezcla } \\
90 \% \text { Arena } \\
\text { Ottawa, 10\% } \\
\text { de Caolinita }\end{array}$} & 300 & 72 & 143 & 29,47 & 12,8 & 16,67 & $56,6 \%$ \\
\hline 5 & & & 1600 & 72 & 143 & 150,47 & 124,1 & 26,33 & $17,5 \%$ \\
\hline 6 & & & 2500 & 72 & 143 & 249,80 & 216,1 & 33,67 & $13,5 \%$ \\
\hline 7 & \multirow{3}{*}{ Fluoresceína } & \multirow{3}{*}{$\begin{array}{c}\text { Mezcla } \\
90 \% \text { Arena } \\
\text { Ottawa, 10\% } \\
\text { de Caolinita }\end{array}$} & 60 & 60 & 143 & 5,87 & 5,6 & 0,24 & $4,13 \%$ \\
\hline 8 & & & 200 & 60 & 143 & 19,93 & 19,5 & 0,41 & $2,04 \%$ \\
\hline 9 & & & 400 & 60 & 143 & 39,72 & 39,2 & 0,55 & $1,39 \%$ \\
\hline 10 & \multirow{3}{*}{ Fluoresceína } & \multirow{3}{*}{$\begin{array}{l}\text { Mezcla 90\% } \\
\text { Brady Sand } \\
\text { 16/30, 10\% } \\
\text { de Caolinita }\end{array}$} & 60 & 60 & 143 & 5,91 & 5,9 & 0,04 & $0,6 \%$ \\
\hline 11 & & & 200 & 60 & 143 & 19,82 & 19,8 & 0,03 & $0,1 \%$ \\
\hline 12 & & & 400 & 60 & 143 & 40,18 & 40,1 & 0,04 & $0,1 \%$ \\
\hline 13 & \multirow{3}{*}{ Eosina $Y$} & \multirow{3}{*}{$\begin{array}{c}\text { Mezcla } \\
90 \% \text { Arena } \\
\text { Ottawa, 10\% } \\
\text { de Caolinita }\end{array}$} & 100 & 60 & 143 & 9,87 & 9,6 & 0,30 & $3,1 \%$ \\
\hline 14 & & & 300 & 60 & 143 & 29,25 & 28,9 & 0,36 & $1,2 \%$ \\
\hline 15 & & & 500 & 60 & 143 & 59,74 & 59,2 & 0,53 & $0,9 \%$ \\
\hline 16 & \multirow{3}{*}{ Eosina $\mathrm{Y}$} & \multirow{3}{*}{$\begin{array}{l}\text { Mezcla 90\% } \\
\text { Brady Sand } \\
\text { 16/30, 10\% } \\
\text { de Caolinita }\end{array}$} & 100 & 60 & 143 & 9,67 & 9,5 & 0,21 & $2,2 \%$ \\
\hline 17 & & & 300 & 60 & 143 & 29,36 & 29,1 & 0,25 & $0,9 \%$ \\
\hline 18 & & & 500 & 60 & 143 & 59,11 & 59,0 & 0,15 & $0,3 \%$ \\
\hline 19 & \multirow{3}{*}{ Tiocianato } & \multirow{3}{*}{$\begin{array}{c}\text { Mezcla } \\
90 \% \text { Arena } \\
\text { Ottawa, 10\% } \\
\text { de Caolinita }\end{array}$} & 100 & 60 & 143 & 9,99 & 9,7 & 0,29 & $2,9 \%$ \\
\hline 20 & & & 400 & 60 & 143 & 39,41 & 38,8 & 0,57 & $1,4 \%$ \\
\hline 21 & & & 750 & 60 & 143 & 69,56 & 69,1 & 0,43 & $0,6 \%$ \\
\hline 22 & \multirow{3}{*}{ Tiocianato } & \multirow{3}{*}{$\begin{array}{l}\text { Mezcla } 90 \% \\
\text { Brady Sand } \\
\text { 16/30, 10\% } \\
\text { de Caolinita }\end{array}$} & 100 & 60 & 143 & 9,99 & 9,8 & 0,14 & $1,4 \%$ \\
\hline 23 & & & 400 & 60 & 143 & 39,41 & 39,1 & 0,29 & $0,7 \%$ \\
\hline 24 & & & 750 & 60 & 143 & 69,56 & 69,1 & 0,43 & $0,6 \%$ \\
\hline
\end{tabular}

Para la fluoresceína, Tiocianato de Potasio y la Eosina $\mathrm{Y}$, los resultados indican una buena recuperación, y la pérdida se puede atribuir a la incertidumbre del experimento, sumado al el error humano en la preparación de las soluciones. Para la fluoresceína las medidas periódicas de los blancos demuestran que las pérdidas por degradación térmica de las disoluciones se pueden considerar despreciables durante las primeras 110 horas. Después se observa una disminución considerable de la concentración, esto puede ocurrir porque la intensidad de la fluorescencia cae con el transcurso del tiempo y la exposición a temperaturas elevadas, ya que existen altas probabilidades de que la molécula excitada sea desactivada. De aquí se concluye que la fluoresceína no puede ser usada en aplicaciones con tiempos de residencia mayores a 5 días.

El Rojo de metilo no cumplió los parámetros establecidos de tener un grado de adsorción máximo por el adsorbato de $5 \%$, necesario para un balance de masa más 
confiable. Para los nitratos no fue posible determinar la adsorción. Entre los factores a los cuales se puede atribuir este comportamiento, está la incompatibilidad del trazador con algunas de las sustancias usadas durante el proceso de detección, así como la turbidez presentada al adicionar la mezcla de minerales en la solución de trazador, lo que indudablemente influencia la medida de absorbancia en la que se basa el método analítico.

\section{RESULTADOS ENSAYOS DE COMPATIBILIDAD}

Los ensayos de compatibilidad entre el fluido de fractura con el crudo del campo Tibú, presentó 100\% separación de fases, con la interface definida, sin presencia de turbidez, precipitados, gradientes de densidad visible, nata o sólidos suspendidos. Solamente se observó un cambio en el aspecto de la tonalidad del fluido, que se atribuye al color que le da el trazador adicionado.

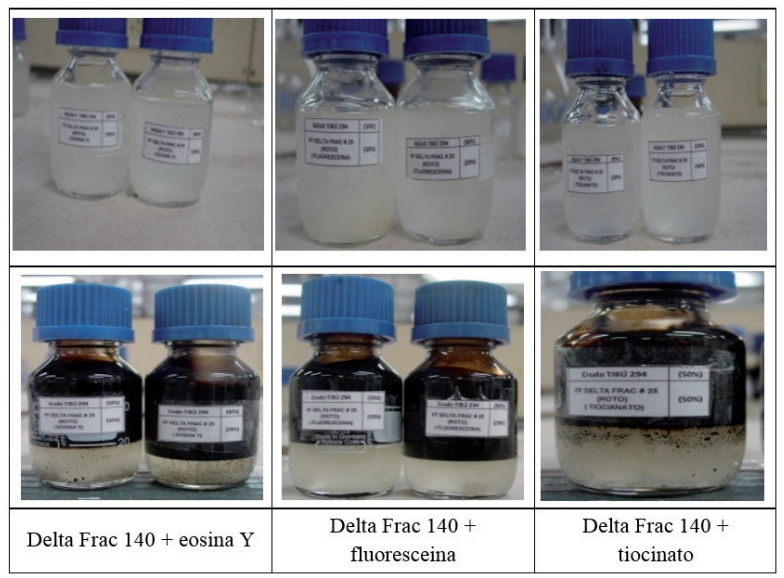

Figura 4. Compatibilidad del fluido de fractura con el crudo y el agua de formación.

Así mismo, durante los ensayos de adsorción del trazador en la fase oleosa, no observa una pérdida significativa ( $2 \%$ y $4 \%)$. Esta reducción puede atribuirse a la degradación fotocatalítica de los trazadores o incluso a la incertidumbre de los ensayos.

Los valores de tensión interfacial obtenidos al adicionar el trazador al FF, se encontraron en el orden de 4-6 dinas/cm. El cambio más evidente se presentó con el fluido marcado con Eosina Y; el cual paso de 5.2 dinas/ $\mathrm{cm}$ que corresponde al valor de tensión del fluido blanco a 4.8 dinas $/ \mathrm{cm}$ para el fluido preparado con el trazador. No obstante, este cambio no afecta las propiedades del fluido, ya que para movilizar el $\mathrm{FF}$ roto se requiere que la tensión interfacial entre el FF y el fluido de formación sea baja con el fin de hacer la recuperación más efectiva y causar el menor daño a la formación.

La Figura 5 presenta el comportamiento reológico del fluido de fractura preparado con cada uno de los trazadores. Se observa que la viscosidad inicial en todos los fluidos es alta, pero presenta una caída vertiginosa hasta un valor aproximado de $700 \mathrm{cP}$ durante los primeros 5 minutos. La formulaciones alcanzan la viscosidad de $100 \mathrm{cP} @ 100 \mathrm{~s}^{-1}$ en un tiempo cercano a las dos horas. El mismo tipo de comportamiento se observó en el fluido de referencia (blanco).

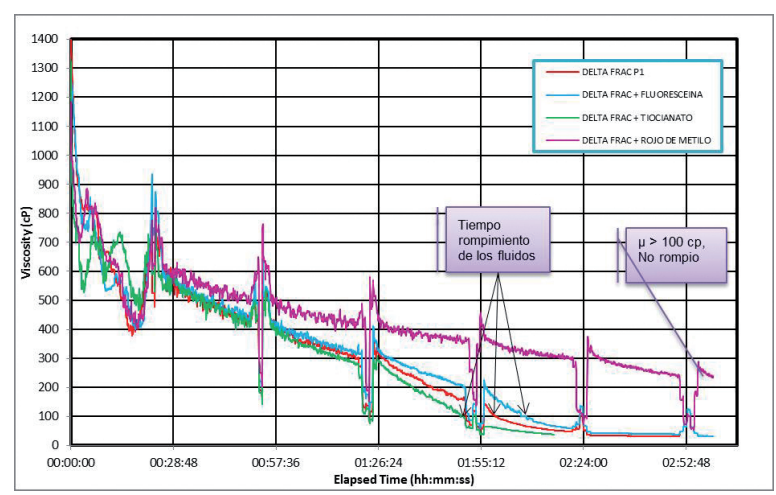

Figura 5. Comparación perfil reológico de los fluidos de fractura.

Después de tres horas, tiempo máximo esperado en esta evaluación, la viscosidad del FF conteniendo con Rojo de metilo como trazador, permaneció por encima de 100 cP, lo cual se interpreta como un rompimiento deficiente del fluido. Esta condición sumada a su alto nivel de adsorción (mayor al 5\%) es suficiente para descartar esta sustancia como trazador.

La viscosidad para la formulación preparada con Eosina Y como trazador, se mantuvo por debajo de la del FF tomado como referencia durante las tres horas de ensayo; y presentó un tiempo de rompimiento de 1:47 horas, que es menor al fluido base ( 2 horas). Sin embargo este cambio no altera de forma significativa las propiedades del fluido.

De acuerdo con los resultados obtenidos en los ensayos de compatibilidad, no se observaron cambios considerables en las propiedades del fluido de fractura causados por las sustancias adicionadas (trazadores) con respeto al fluido tomado como referencia. 


\section{RESULTADOS ENSAYOS DE DESPLAZAMIENTO DEL FLUIDO DE FRACTURA EN UN MEDIO POROSO}

Con el objetivo de evaluar la reducción de la conductividad de las fracturas causadas por la retención del fluido de fractura usando los trazadores químicos seleccionados hasta esta etapa (fluoresceína, Tiocianato de Potasio y Eosina Y) se realizaron tres ensayos de desplazamiento en medios porosos. Para ello, se prepararon muestras de FF dosificado con uno o dos trazadores seleccionados como se describe en la Tabla 1.

Tabla 1. Ensayos de desplazamientos en medios porosos

\begin{tabular}{|ccccc}
\hline $\begin{array}{c}\text { \# de ensayo } \\
\text { (Berea) }\end{array}$ & Trazador & $\begin{array}{c}\text { [ ] } \\
\text { Trazador }\end{array}$ & $\begin{array}{c}\text { VP } \\
\text { Berea }\end{array}$ & $\begin{array}{c}\text { Ka } \\
\text { Berea }\end{array}$ \\
\hline 1 & FLU/TIO & $4,5 / 50$ & 19,9 & 51,63 \\
\hline 2 & EOS/TIO & $4,5 / 50$ & 23,3 & 56,41 \\
\hline 3 & FLU & 4,5 & 23,3 & 65,63 \\
\hline
\end{tabular}

En la Figura 6 se puede apreciar los resultados obtenidos del primer ensayo, que se resumen en la Tabla 2. La recuperación del FF calculada con base en la cuantificación del polímero recuperado fue alrededor del $62,2 \%$ del total inyectado. El FF sobrante fue retenido en el medio poroso y esto produjo la disminución en la permeabilidad inicial del $51 \%$ en los diferentes sistemas.

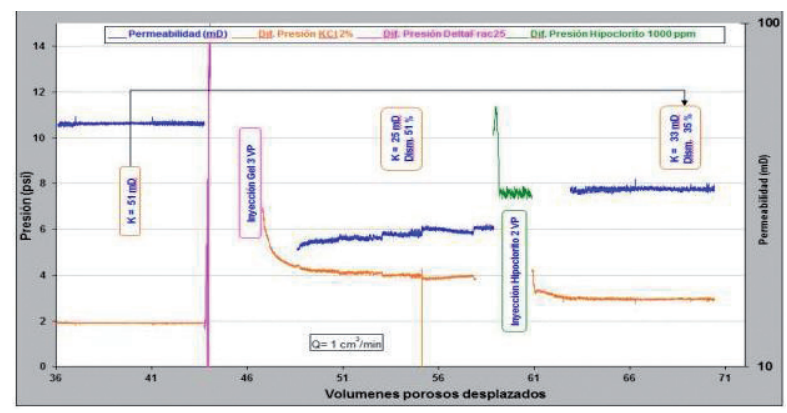

Figura 6. Evaluación en condiciones Dinámicas - Fluido Delta Frac 25 - Trazador Fluoresceina + Tiocianato Berea \# 1.

El mismo cálculo basado en la cuantificación de los dos trazadores adicionados (valores equivalentes a la eficiencia de flowback) fueron $50.8 \%$ para la fluoresceína y del 51,6\% para el Tiocianato de potasio. Valores muy cercanos al porcentaje de disminución en la permeabilidad (51\%) obtenido en el ensayo. El valor estimado con la concentración de polímero fue un $11 \%$ más alto con respecto al valor obtenido con los trazadores, corroborando que los cálculos de la eficiencia de flowback por el método de cuantificación de polímero son superiores al real, lo cual proveería información equivocada de la limpieza de la fractura.
Tabla 2. Porcentaje recuperado de los trazadores y polímero del fluido de fractura en los efluentes de la Berea 1.

\begin{tabular}{|c|c|c|c|c|}
\hline \multirow[b]{2}{*}{ Retornos } & \multicolumn{4}{|c|}{ BEREA 1. FLUORESCEINA/TIOCIANATO } \\
\hline & $\begin{array}{c}\text { Polímero } \\
(\%)\end{array}$ & $\begin{array}{c}\text { Fluoresceína } \\
(\%)\end{array}$ & $\begin{array}{c}\text { Tiocianato } \\
(\%)\end{array}$ & $\begin{array}{c}\text { K } \\
\text { Retenida } \\
(\%)\end{array}$ \\
\hline FF & 62,2 & 50,8 & 51,6 & 49,02 \\
\hline Pos- Frac & 15,04 & & & 64,71 \\
\hline Total & 77,25 & & & \\
\hline
\end{tabular}

La aplicación del tratamiento pos-fractura, posterior a la recuperación del fluido por inyección de salmuera, produjo la recuperación del $15 \%$ del total del polímero inyectado, equivalente al 39,8\% del polímero retenido en el sistema poroso. Esta remoción del polímero implicó la recuperación de la permeabilidad retenida hasta $64,7 \%$ respecto al valor inicial de permeabilidad, es decir se obtuvo un incremento en la permeabilidad del $35 \%$ respecto al valor de permeabilidad obtenida después de desplazar el fluido de fractura.

De acuerdo con la Figura 7, en los efluentes se detalla que el primer trazador (Fluoresceína) fue más afín con lo que se esperaba, con un comportamiento estable, con concentración similar en cada volumen poroso recolectado, mientras la concentración del Tiocianato de potasio presenta una mayor variación. Este comportamiento del tiocianato podría sugerir que existe una auto-concentración del trazador en la Berea, sin embargo, en este ensayo no es un factor relevante, dado que las diferencias en la concentración no son muy significativas. (Varían entre 1 y 2\%).

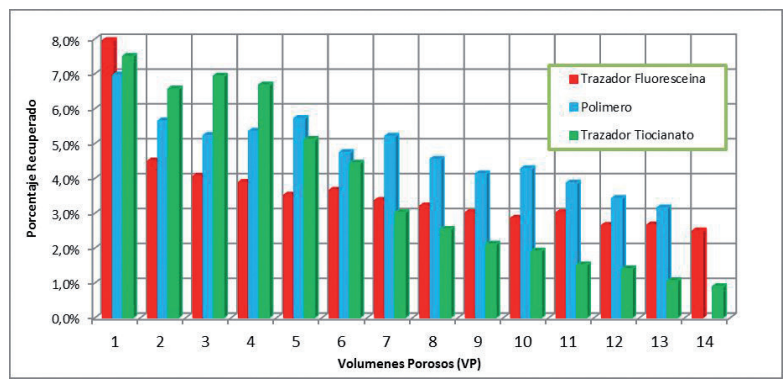

Figura 7. Porcentaje de trazador y polímero recuperado en los efluentes Berea 1

Los resultados indican que los trazadores evaluados en esta Berea, pueden ser usados para determinar la eficiencia de recuperación de fluido de fractura, obteniéndose resultados que concuerdan con la disminución en la permeabilidad obtenida, con porcentajes similares para ambos trazadores.

En la segunda muestra de Berea se evalúo la efectividad de la Eosina Y junto con el Tiocianato de potasio para 
determinar la eficiencia de Flowback. (Figura 8) En este caso, el polímero retenido causó una disminución en la permeabilidad del $51 \%$ con respecto a la permeabilidad inicial dejando una permeabilidad retenida del $48,9 \%$.

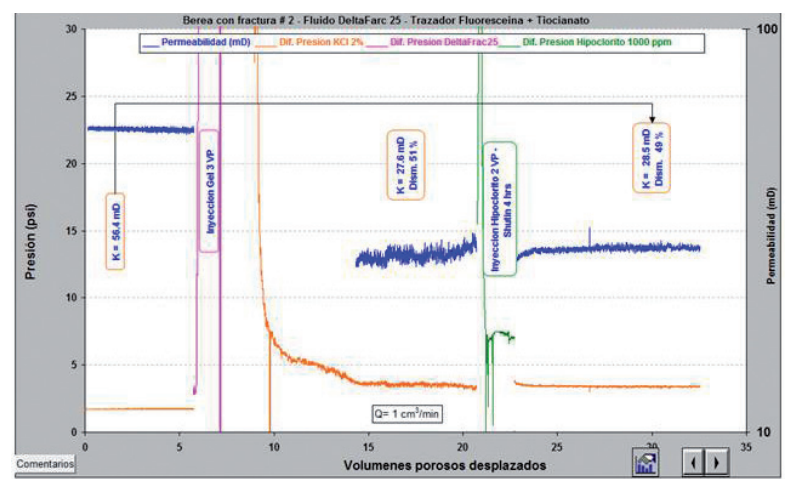

Figura 8. Evaluación en condiciones Dinámicas - Fluido DeltaFrac 25 - Trazador Eosina Y + Tiocianato Berea \# 2.

Se obtuvo un porcentaje del $69,74 \%$ de FF recuperado calculado por el método del polímero medido en los efluentes. Al igual que en el ensayo anterior los cálculos de la eficiencia de "flowback" calculada con base en la cuantificación del polímero fue mayor en 14,29\% con respecto al Tiocianato de potasio y $21,91 \%$ respecto a la Eosina Y.

Mientras que por cuantificación de los trazadores, los resultados fueron de 55,4\% para Tiocianato de potasio y de $47,8 \%$ para la Eosina Y respectivamente. Valores que son más coherentes con la disminución en la permeabilidad del $51 \%$ causada por el polímero retenido. En consecuencia, se ratifica que los cálculos de la eficiencia de flowback demuestran que los trazadores proporcionan resultados más acertados para determinar la eficiencia de recuperación del fluido de fractura.

El tratamiento pos-frac dio como resultado un bajo incremento en la permeabilidad, pasando de $27,6 \mathrm{mD}$ a $28,5 \mathrm{mD}$; promoviendo la recuperación de apenas el $7,5 \%$ del total del polímero inyectado, equivalente al $24,8 \%$ del polímero retenido en el sistema poroso. Esta recuperación del polímero causó recuperación en la permeabilidad retenida hasta el 50,5\%.

De acuerdo con la Figura 9, las muestras de efluentes o flowback recolectados en este ensayo no contienen una concentración de polímero uniforme. Este comportamiento se puede atribuir a que mientras se genera el "cake" pasa una cantidad considerable de fluido por la muestra, en la cual la concentración del polímero en los primeros instantes es menor y una vez que se genera el "cake” la concentración del polímero puede aumentar, luego empieza a verse una pérdida de fluido, que corresponde al filtrado, dando lugar a una reducción en la concentración del polímero en el fluido filtrado ó efluente. Por el contrario, no hay evidencia del mismo impacto por formación de cake para los trazadores.

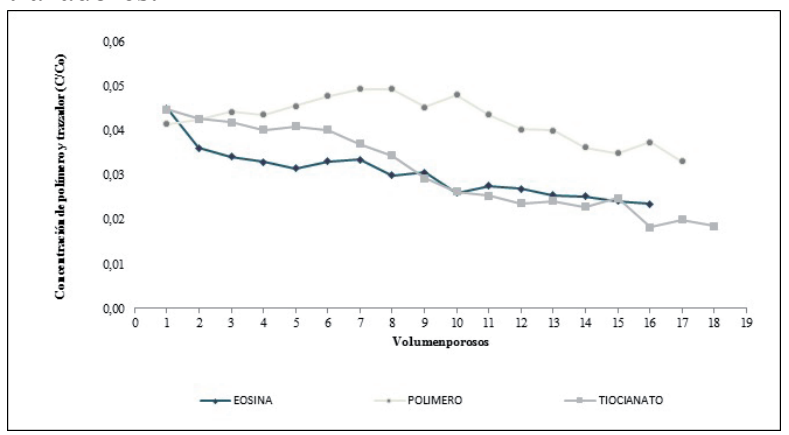

Figura 9. Concentración de Polímero y Trazadores en los Efluentes Berea.

Finalmente, para cuantificar la concentración de los trazadores en los retornos colectados después de la aplicación del tratamiento pos fractura y la cantidad de trazador retenido en la berea se realizó el ensayo de desplazar el FF preparado con un solo trazador (Fluoresceína) a través de la muestra de berea. Figura 10.

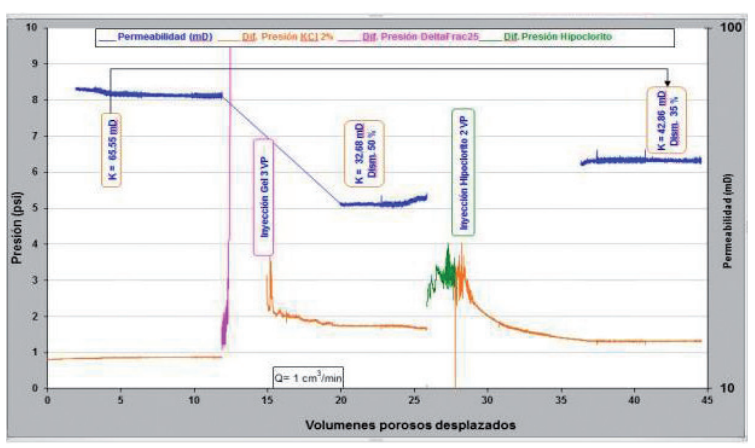

Figura 10: Evaluación en condiciones Dinámicas - Fluido Delta Frac 25 - Trazador Fluoresceina Berea \# 3

En la Tabla 3 se observa que el cálculo de la recuperación del FF usando la concentración de polímero medida en los efluentes, en la primera etapa (desplazamiento del FF mediante KCL) fue del $57,1 \%$ del total inyectado y del $47,8 \%$ usando la concentración medida del trazador. El fluido retenido causó una disminución de la permeabilidad inicial del $50 \%$.

Después de la aplicación del tratamiento pos frac (Etapa 2) el porcentaje recuperado del FF según la medición de la concentración polímero fue del $17 \%$ y medido con el trazador el porcentaje recuperado fue del $15,3 \%$. Se debe tener en cuenta que de acuerdo con los resultados obtenidos en los ensayos de compatibilidad del 
trazador con el tratamiento pos-fractura, la lectura en la absorbancia del trazador se reduce en cerca del 10\% por la degradación causada por el tratamiento, luego el porcentaje de trazador recuperado en la segunda etapa sería de $16,8 \%$.

Tabla 3. Recuperación de los trazadores - polímero del fluido de fractura Berea.

\begin{tabular}{lccc|} 
& \multicolumn{3}{c|}{ BEREA 3. FLUORESCEINA } \\
\hline Retornos & $\begin{array}{c}\text { Polímero } \\
\mathbf{( \% )}\end{array}$ & $\begin{array}{c}\text { Fluoresceína } \\
\mathbf{( \% )}\end{array}$ & $\begin{array}{c}\text { K } \\
\text { Retenida } \\
(\%)\end{array}$ \\
\hline FF & 57,1 & 47,8 & 48,9 \\
\hline Pos- Frac & 17 & 16,8 & 65,4 \\
\hline $\begin{array}{l}\text { Solución de } \\
\text { Hipoclorito 1 }\end{array}$ & 15 & 20,6 & \\
\hline $\begin{array}{l}\text { Solución de } \\
\text { Hipoclorito 2 }\end{array}$ & 1,7 & 0 & \\
Total & 91,7 & 85,2 & \\
\hline
\end{tabular}

La eficiencia de recuperación del flowback en la primera etapa, calculada mediante el polímero recuperado es del $57,1 \%$. De acuerdo con estos resultados y si la reducción en la permeabilidad fuese directamente proporcional al polímero retenido, se podría afirmar que existe una reducción en la permeabilidad del $43 \%$. Sin embargo, el polímero retenido en el medio poroso causó una disminución en la permeabilidad del $50 \%$, pasando $65,55 \mathrm{mD}$ a $32,68 \mathrm{mD}$.

La eficiencia de flowback calculada mediante el polímero recuperado da como resultados un porcentaje superior en 7\%. Con lo cual se puede indicar que la cantidad de polímero recuperado en los efluentes no es proporcional al retorno permeabilidad del sistema poroso. Mientras que, por medio del trazador se obtuvo un valor de $47,8 \%$, valor más cercano a la pérdida de permeabilidad obtenida, validando que la eficiencia de flowback calculada por medio de los trazadores tienen mayor coherencia.

En la primera etapa alcanza una recuperación del 47,8\%, valor cercano a la reducción por la permeabilidad (50\%). Así mismo, un 37,3\% del trazador es recuperado en tratamientos posteriores, con lo cual el porcentaje total recuperado llega al $85,1 \%$. Mientras, el polímero recuperado en la primera etapa es del $57,1 \%$ valor que se aleja en mayor grado a la reducción de la permeabilidad obtenida. El polímero total recuperado asciende al $91,7 \%$.

Los resultados obtenidos, permiten afirmar que, bajo el margen de incertidumbre asociado a estos ensayos, los trazadores fluoresceína, Tiocianato y Eosina Y son técnicamente efectivos para determinar la eficiencia de flowback durante un proceso de fracturamiento hidráulico, ya que permiten obtener resultados que describen la reducción de la permeabilidad causada por el fluido de fractura retenido. Además cabe destacar, que se obtienen valores similares con los distintos trazadores durante este los ensayos.

\section{CONCLUSIONES}

Entre las alternativas planteadas como trazadores en este trabajo, se identificaron tres sustancias (fluoresceina, tiocianatos y eosina Y) que superan el protocolo experimental propuesto para evaluarlas como trazadores en un fluido de fractura.

Debido al grado de descomposición térmica de la fluoresceína, su uso como trazador en procesos de fracturamiento hidráulico se debe limitar a yacimientos de temperatura media (hasta $180^{\circ} \mathrm{F}$ ) y periodos de tiempo cortos, debido a que se observa una disminución considerable de la concentración causados por este efecto.

La incompatibilidad del rojo de metilo con el fluido de fractura, especialmente en su comportamiento reológico, así como la alta adsorción presentada por parte del rojo de fenol en el medio poroso, descarta su uso como trazadores un fluido de fractura base agua tipo goma guar.

Las sales de nitrato de potasio y nitrato de sodio durante los ensayos evidenciaron comportamientos no esperados como trazadores, que merecen un mayor análisis a fin de obtener interpretaciones fidedignas.

Dado que los trazadores no se auto-concentran proporcionan información más confiable sobre la eficiencia de retorno del fluido de fractura que los análisis realizados con la concentración de polímero. Este hecho constituye en una ventaja para para su uso.

Los cálculos de la cantidad de fluido retornado mediante el polímero recuperado son más altos que en la realidad, lo que podría proporcionar información engañosa sobre la limpieza de la fractura.

\section{NOMENCLATURA}

IP: Índice de productividad

FF: $\quad$ Fluido de fractura

ppb: Partes por billón

cP : $\quad$ Centipoise

$\mathrm{T}_{\mathrm{y}}$ : Temperatura del yacimiento. 
rpm: $\quad$ Revoluciones por minuto

$\mathrm{mL}$ : mililitros

min: $\quad$ minutos

SP: $\quad$ Sentido de producción.

SI. Sentido de inyección.

VP: $\quad$ Volumen poroso

Ka Permeabilidad absoluta

Q. Caudal

QA/QC: Quality Assurance/Quality Control

\section{BIBLIOGRAFÍA}

1. Agenet, N., Tehrani, N.M., Tillemnet, T.O. "Fluorescent Nanobeads: A new Generation of Easily Detectable Water Tracers". Paper IPTC 15312 presented at the International Petroleum Technology conference, Bangkok, Thailand, 7 -9 February 2012.

2. Ahma, S., Basak, K., and Hossein, K. "Analysis of Chemical tracer Flowback in Unconvectional Reservoirs". Paper SPE -171656-MS presented at the SPE/SCUR Unconvectional Resource. Alberta Canada, 30 September - 2 October. 2014.

3. Asadi, M., Woodroof, R. "Comparative Study of Flowback Analysis Using Polymer Concentrations and Fracturing Fluid Tracer Methods": A Field Study. SPE Journal Paper No 101614 December 2006.

4. Asadi, M., Woodroof, R. William S. "Monitoring Fracturing Fluid Flowback with Chemical Tracers: A Field Case Study". Paper SPE 77750 presented at the SPE Annual Technical Conference and Exhibition held in San Antonio, Texas, 29 September-2 October 2002.

5. Asadi, M., WoodrooF, Robert A., and Dumas, Donald Jr. "Post-Frac Analysis Based on Flowbacks Results Using Chemical Frac-Tracers". IPTC International Petroleum Technology Conference Paper No 11891 December 2008.

6. Carrascal, F. A., Pachón, Z. \& Molina, D. R. "Post-fracturing treatments design to redress the damage caused by fracturing fluid polymers". CT\&F - Ciencia, Tecnología y Futuro, 2014.

7. Du, Y., and Guan, L. "Interwell Tracers Test: Lesson Learned From Past Field Studies". Papers SPE 93140 presented at the Asia Pacific Oil \& Gas Conference and Exhibition, Jakarta, 5 - 7 April. 2005.

8. Hutchins, R.D., and Dovan, H.T. "Aqueous Tracer for Oilfield Applications". Papers SPE 21049 presented at the SPE International Symposium on Oilfield Chemistry, Anaheim, 20 - 22 February 1991.

9. Mcintyre, F.J. Polkoswski, G.A., Pow, M.J. "Radioactive Tracers Monitor Solvents Spreading in Rainbow Vertical Hydrocarbon Miscible Flood". SPE Reservoir Engineering. February 1998.

10. Melo, M.A., Holleben, C.R., and Almeida, A.R. "Using Tracers to Characterize Petroleum Reservoirs: Application to Carmopolis Field, Brazil". Paper SPE 69474, presentation at the SPE Latin American and Caribbean Petroleum Engineering Conference held in Buenos Aires, Argentina, 25-28 March 2001.

11. Pope, D., et al. "Field study of guar removal from hydraulic fractures". Paper SPE 31094 presented at the SPE International Symposium on Formation Damage Control. Lafayette, La, Feb. 14-15. 1995.

12. Reinicke A., Rybacki E., Stanchits S., Huenges E. and Dresen G. "Hydraulic fracturing stimulation techniques and formation damage mechanismImplications from laboratory testing of tight sanstone- proppanyt system". Chemieder Erde, 2010 .

13. Shan, S.N, Lord D.L, and Tan, HC. "Recent advances in the fluid mechanics and the fluid rheology of fracturing fluid". EN: SPE Journal paper N ${ }^{\circ} 22391$ March, 1992.

14. Sullivan, R., et al. "Optimizing Fracturing Fluid Cleanup in the Bossier Sand Using Chemical Frac Tracers and Agressive Gel Breaker Deployment". SPE Journal Paper No 90030 September 2004.

15. Wang, J., Holditch, S. and McVay., D. "Effect on Gel Damage on Fractures Fluid Cleanup and Long Term Recovery in Tight Gas Reservoirs". Journal of Natural Gas science and Engineering 9, 2012.

16. Wheeler V., Parsons T., "The Application of Radioactive Tracers to Oil Reservoir Water floods Studies". SPE 13985/1. 1985.

17. Yang, B. H. and Flippen, M. C. "Improved flowback analysis to asses polymer damage". Paper SPE 37444 presented at the SPE Production Operations Symposium, Oklahoma City, OK, March 9-11. 1997.

Recepción: 15 de Julio de 2015

Aceptación: 24 de Octubre de 2015 